\title{
New technique for molecular-dynamics computer simulations: Hellmann-Feynman theorem and subspace Hamiltonian approach
}

\author{
Madhu Menon and Roland E. Allen \\ Department of Physics, Texas A\&M University, College Station, Texas 77843-4242
}

(Received 26 August 1985)

\begin{abstract}
In the past, molecular-dynamics computer simulations have employed model potentials of the interaction between atoms. Here we introduce a new molecular-dynamics technique in which atomic forces are computed from the total electronic energy of the system of interacting atoms. The method is illustrated by trajectories for Al and As atoms scattering off the relaxed (110) surface of GaAs.
\end{abstract}

\section{INTRODUCTION}

Many important physical and chemical phenomena involve molecules and atoms interacting with solid surfaces. A technique for realistic simulation of molecular motions at surfaces would be of great utility, both for obtaining fundamental understanding of such processes and for allowing simulation of systems of technological interest. Potential applications include catalysis and the growth of advanced materials such as superlattices. Also, interactions of incident metal atoms with semiconductor surfaces are of interest in conjunction with the formation of semiconductor-metal contacts. ${ }^{1-5}$ In the past, computer simulations of surfaces and of atom-surface interactions have been treated using Lennard-Jones potentials. ${ }^{6-8}$ This approach, although giving interesting qualitative results, fails to provide a quantitative description of covalently-bonded materials, such as the III-V compound and group-IV elemental semiconductors.

In this paper, we introduce a technique employing the Hellmann-Feynman theorem and certain new Green'sfunction methods that are described below. In the present calculations we obtain the required Green's functions by approximating the electronic structure of the system with a semiempirical tight-binding Hamiltonian. ${ }^{\text {? }}$

\section{FORMALISM}

The force on each atom is calculated using Green'sfunction techniques. In the present application, we illustrate the general technique by treating the special case of a single atom coming in on a GaAs (110) surface, with only this incident atom allowed to move. We first obtain the GaAs surface Green's function, $G_{s}$, using a method similar to that of Kalkstein and Soven. ${ }^{10}$ The subspace of the perturbation is then made larger by including the unperturbed Green's function of the incident atom $A$ :

$$
G_{0}=\left(\begin{array}{cc}
G_{A} & 0 \\
0 & G_{s}
\end{array}\right),
$$

where $G_{0}$ is the unperturbed (noninteracting) Green's function of the GaAs surface plus the atom $A$.

The interaction between the incident atom and the sur- face is introduced through a perturbation, $V$, with matrix elements only between $\mathrm{Ga}$ and $A$ and $\mathrm{As}$ and $A$. These matrix elements represent covalent-bonding interactions. One can then obtain a Green's function, $G$, including the interactions, from $G_{0}$ and $V$ via the one-electron Dyson equation

$$
G=G_{0}+G_{0} V G \text {. }
$$

The energy of the system $E$ is given by summing the one-electron energies $\epsilon$ :

$$
E=\int_{-\infty}^{\epsilon_{F}} d \epsilon \epsilon \rho(\epsilon)
$$

where

$$
\rho(\epsilon)=-\frac{1}{\pi} \operatorname{Im} \operatorname{Tr} G(\epsilon)
$$

is the density of states. Double-counting of the Coulomb interaction is approximately treated via the repulsive interaction introduced below. Calculations for larger subspaces (which allow recoil of the surface atoms, formation of surface defects, etc.) can be treated with the same technique and are now in progress.

The force is found by evaluating the change in the energy $E$ of (3) due to a change in the position of the atom $A$. Suppose that we make a small change in the position, say, in the $x$ direction. The change in the energy of the system, $\Delta E$, is then

$$
\Delta E=\int_{-\infty}^{\epsilon} d \epsilon \epsilon \Delta \rho(\epsilon),
$$

where $^{11}$

$$
\Delta \rho=-\frac{1}{\pi} \frac{\partial}{\partial \epsilon} \operatorname{Im} \ln \operatorname{det}(1-G \Delta H),
$$

and $\Delta H$ is the change in the Hamiltonian when $x \rightarrow x+\Delta x$.

One can expand the determinant to first order in $\Delta H$. The result is

$$
\operatorname{det}(1-G \Delta H)=1-\operatorname{Tr}(G \Delta H) .
$$

Taking the logarithm, keeping terms of order $\Delta H$ only, using (4) and (5), and integrating by parts, we obtain 


$$
\Delta E=-\frac{1}{\pi} \operatorname{Im} \operatorname{Tr} \int_{-\infty}^{\epsilon_{F}} d \epsilon G \Delta H .
$$

From the definition of force,

$$
F_{x}=-\lim _{\Delta x \rightarrow 0} \frac{\Delta E}{\Delta x},
$$

we then obtain

$$
F_{x}=\frac{1}{\pi} \operatorname{Im} \operatorname{Tr} \int_{-\infty}^{\epsilon_{F}} d \epsilon G \frac{\partial V}{\partial x},
$$

where

$$
H=H_{0}+V \text {. }
$$

We evaluate the Green's function $G$ using the subspace Hamiltonian approach, described in detail elsewhere. ${ }^{12}$ Briefly, if $H_{\text {sub }}(\epsilon)$ is defined by

$$
\epsilon-H_{\text {sub }}(\epsilon)=\left[G_{0}(\epsilon)\right]^{-1}-V,
$$

then the Green's function has the representation

$$
G(\epsilon)=\sum_{i} \frac{\psi_{i}(\epsilon) \bar{\psi}_{i}^{\dagger}(\epsilon)}{\epsilon-\epsilon_{i}(\epsilon)},
$$

where

$$
H_{\mathrm{sub}}(\epsilon) \psi_{i}(\epsilon)=\epsilon_{i}(\epsilon) \psi_{i}(\epsilon)
$$

and

$$
H_{\mathrm{sub}}^{\dagger}(\epsilon) \bar{\psi}_{i}(\epsilon)=\epsilon_{i}^{*}(\epsilon) \bar{\psi}_{i}(\epsilon)
$$

$H_{\text {sub }}(\epsilon)$ is non-Hermitian if $\epsilon$ is complex. It is also energy dependent. However, for $\epsilon$ complex and well removed from the real axis, we find that $H_{\text {sub }}$, its eigenvalues, and its eigenvectors vary only slowly with $\epsilon .^{12}$ This is demonstrated in Fig. 1. We therefore can calculate the integral of (8) analytically.

The result is

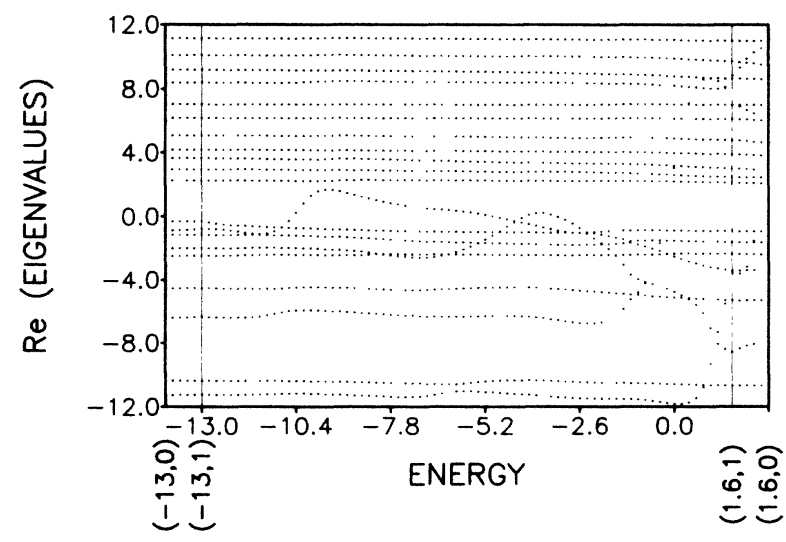

FIG. 1. Eigenvalues $\epsilon_{i}(\epsilon)$ of subspace Hamiltonian as functions of energy $\epsilon$, with $\epsilon$ complex. The results shown are for the rectangular contour $\operatorname{Re} \epsilon=-13.0 \mathrm{eV}$ and $0 \leq \operatorname{Im} \epsilon \leq 1.0 \mathrm{eV}$; $\operatorname{Im} \epsilon=1.0 \mathrm{eV}$ and $-13.0 \mathrm{eV} \leq \operatorname{Re} \epsilon \leq 1.6 \mathrm{eV}$; and $\operatorname{Re} \epsilon=1.6 \mathrm{eV}$ and $1.0 \mathrm{eV} \geq \operatorname{Im} \epsilon \geq 0$. Notice that $\epsilon_{i}(\epsilon)$ is a rather slowly varying function of $\epsilon$ in the complex plane. This is why Eqs. (8) and (10) can be approximated by Eq. (11).
$F_{x}=\frac{1}{\pi} \operatorname{Im} \operatorname{Tr} \sum_{i} \psi_{i}\left(\epsilon_{0}\right) \bar{\psi}_{i}^{\dagger}\left(\epsilon_{0}\right) \ln \left(\frac{\epsilon_{F}-\epsilon_{i}\left(\epsilon_{0}\right)}{\epsilon_{c}-\epsilon_{i}\left(\epsilon_{0}\right)}\right) \frac{\partial V}{\partial x}$.

Here, $\epsilon_{c}$ is a cutoff energy (chosen to be $-12 \mathrm{eV}$ in the present calculation, since this energy lies just below the $s p^{3}$ valence bands of the GaAs). Notice that $F_{x}$ is insensitive to the precise value of $\epsilon_{c}$, because of the logarithmic dependence. Also, $\epsilon_{0}$ is a complex energy [chosen to be $(-6.0 \mathrm{eV}, 1.0 \mathrm{eV})$ in the present calculation]. The philosophy of approximating (8) and (10) by (11), and its justification, are given in Ref. 12.

There are two further points. The surface Green's function of (1) represents an integral over planar wave vectors $\bar{k}=\left(k_{1}, k_{2}\right)$ :

$$
G_{s}=\int d \bar{k} G_{s}(\bar{k}),
$$

where $\int d \bar{k}$ is over the surface Brillouin zone. We approximate this integral with the use of a single special point in the irreducible element; in the present context, this is a rather good approximation. Also, the force of (11) is only the attractive bonding force. There is also a repulsive force, ${ }^{13}$ which we model through a potential $A / r^{n}$. In the present illustrative calculations we took $n=6$.
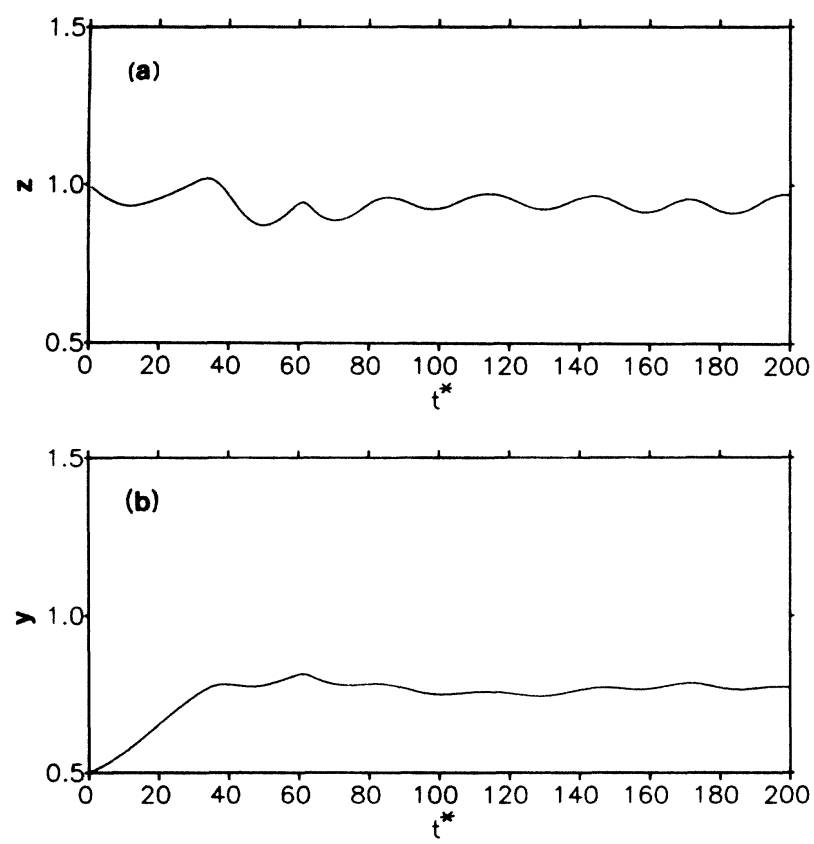

FIG. 2. (a) The $z$ coordinate of an $\mathrm{Al}$ atom incident on a relaxed $\mathrm{GaAs}$ (110) surface as a function of time. (The surface is in the $x-y$ plane.) The perpendicular component of the initial velocity is $-9.5 \times 10^{2} \mathrm{~m} / \mathrm{sec}$ and the parallel component is $9.5 \times 10^{2} \mathrm{~m} / \mathrm{sec}$, in the positive $y$ direction. The time step $\Delta t$ is $5.3 \times 10^{-14} \mathrm{sec}$. The initial coordinates of the incident $\mathrm{Al}$ atom are $(0,0.5 \AA, 1.0 \AA)$, with a Ga substrate atom at $(0,0,0)$ and the $y$ axis pointing from a $\mathrm{Ga}$ atom to an As substrate atom. The time scaled by the "natural" time unit, $5.3 \times 10^{-12} \mathrm{sec}$, is called $t^{*}$. (b) The $y$ coordinate as a function of time for the same $\mathrm{Al}$ atom. 

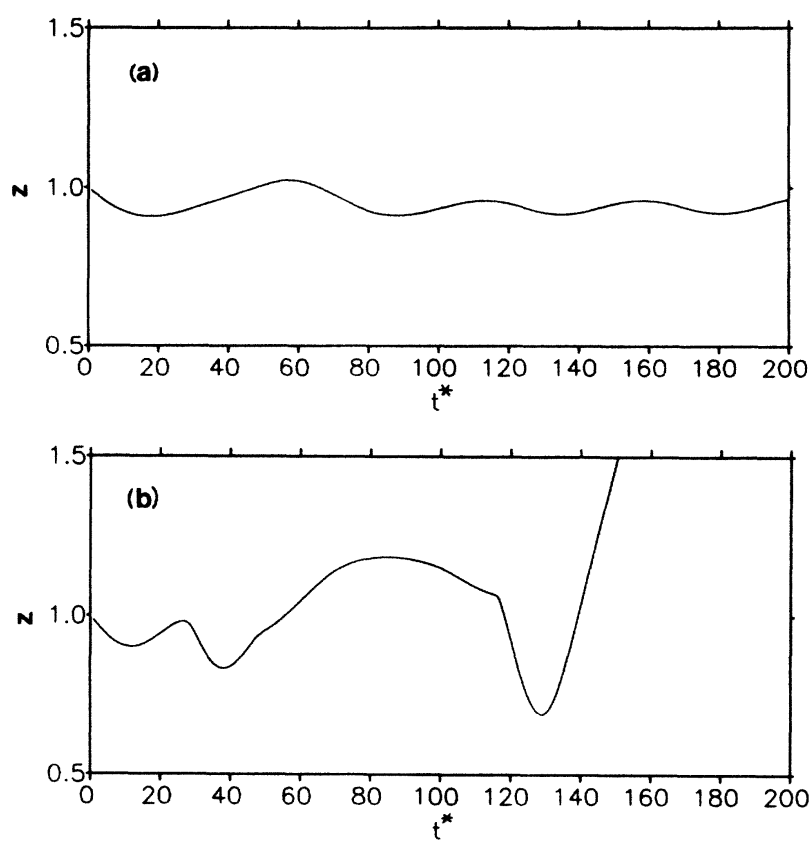

FIG. 3. (a) The $z$ coordinate of an incoming As atom, with the same initial conditions as the $\mathrm{Al}$ atom of Fig. 2, as a function of time. (b) Both the perpendicular and parallel components of the velocity of the $\mathrm{Al}$ atom of Fig. 2 are doubled (to $\left.1.9 \times 10^{3} \mathrm{~m} / \mathrm{sec}\right)$. The $z$ coordinate is plotted as a function of the time.

\section{RESULTS}

The force equation is integrated to obtain the coordinates of the incoming atom $A$ as a function of time $t$. We take the $x-y$ plane to be the plane of the GaAs surface. The $z$ coordinate is positive above the surface. We use the following "natural" units: length, $1 \AA$; force, $1 \mathrm{eV} / \AA$; time, $5.3 \times 10^{-12} \mathrm{sec}$; and velocity, $1.9 \times 10^{3} \mathrm{~m} / \mathrm{sec}$. For all of the results shown below, we damp the motion of the incident atom $A$ to simulate energy loss to the substrate.
(Each time step, 5\% of its kinetic energy is removed.) Damping is turned off after 100 time steps.

In Fig. 2(a), we plot the $z$ coordinate of an incident $\mathrm{Al}$ atom as a function of time. We see that it comes near the surface and settles, oscillating about the energy minimum. Figure 2(b) shows similar action in the $y$ direction. The atom settles into a position between the $\mathrm{Ga}$ and As substrate atoms. Figure 3(a) shows the analogous results for an incident As atom (which is 2.8 times heavier, so that the oscillations are slower). When the kinetic energy of the incident atom is increased, the atom does not get trapped on the surface but escapes, as can be seen in Fig. 3(b).

\section{CONCLUSION}

We introduce a new technique for molecular-dynamics computer simulations, based on the Hellmann-Feynman theorem and certain Green's-function methods that we have developed. This technique is illustrated by preliminary simple calculations for $\mathrm{Al}$ and As atoms incident on the relaxed (110) surface of GaAs. The technique permits, for the first time, simulation of the motion of molecules and atoms in real, covalently-bonded systems. We think that this technique has great promise for studies of the growth of semiconductor-solid and other interfaces, the diffusion and reaction of molecules on catalyst surfaces, and other processes of fundamental and technological interest. Although the present exemplary calculations involve a semiempirical model of the electronic structure, the method is in principle exact-even with many-body effects included-as the available computer time and memory become arbitrarily large, since it is based on rather general Green's function methods.

\section{ACKNOWLEDGMENTS}

We thank the U.S. Office of Naval Research for its support, which made this work possible through Grant No. N00014-82-K-0447. Additional support was provided by the Robert A. Welch Foundation.
${ }^{1}$ W. E. Spicer, P. W. Chye, P. R. Skeath, C. Y. Su, and I. Lindau, J. Vac. Sci. Technol. 16, 1422 (1979).

${ }^{2}$ W. E. Spicer, I. Lindau, P. R. Skeath, and C. Y. Su, J. Vac. Sci. Technol. 17, 1019 (1980).

${ }^{3}$ W. Mönch, Surf. Sci. 132, 92 (1983), and references therein.

${ }^{4}$ R. H. Williams, Surf. Sci. 132, 122 (1983), and references therein.

${ }^{5}$ H. H. Wieder, Inst. Phys. Conf. Ser. 50, 234 (1980); Appl. Phys. Lett. 38, 170 (1980).

${ }^{6}$ R. E. Allen, F. W. de Wette, and A. Rahman Phys. Rev. 179, 887 (1969).
7J. C. Tully, Surf. Sci. 125, 282 (1983).

${ }^{8}$ F. F. Abraham, J. Vac. Sci. Technol. B 2, 534 (1984), and references therein.

${ }^{9}$ P. Vogl. H. P. Hjalmarson, and J. D. Dow, J. Phys. Chem. Solids 44, 365 (1983).

${ }^{10}$ D. Kalkstein and P. Soven, Surf. Sci. 26, 85 (1971).

${ }^{11}$ T. L. Einstein and J. R. Schrieffer, Phys. Rev. B 7, 3629 (1973).

${ }^{12}$ R. E. Allen and M. Menon, Phys. Rev. B (to be published).

${ }^{13}$ See, e.g., W. A. Harrison, Electronic Structure and the Properties of Solids (Freeman, San Franscisco, 1980). 